\title{
An efficient method for LEED crystallography
}

\author{
G. Kleinle ${ }^{\mathrm{a}}$, W. Moritz ${ }^{\mathrm{b}}$ and G. Ertl ${ }^{\mathrm{a}}$ \\ ${ }^{a}$ Fritz-Haber-Institut der Max-Planck-Gesellschaft, Faradayweg 4-6, D-1000 Berlin 33, Germany \\ ${ }^{b}$ Institut für Kristallographie und Mineralogie, Universität München, Theresienstrasse 41, 8000 München 2, Fed. Rep. of Germany
}

Received 7 May 1990; accepted for publication 2 July 1990

\begin{abstract}
Determination of periodic surface structures from analysis of LEED intensity data is usually based on the evaluation of continuous $I / V$-spectra for a large number of model structures for which all the structural parameters have to be refined simultaneously until the best agreement with the experimental data, as quantified by the minimum of $R$-factor, is achieved. It is demonstrated that analysis based on intensity data taken only at discrete energy intervals (of up to about $20 \mathrm{eV}$ ) leads to no loss in accuracy if compared with the evaluation of continuous $I / V$-spectra. The introduction of a novel $R_{\mathrm{DE}}$-factor permits in addition to replace the "grid search" technique by a "least-squares" optimisation scheme which enables automatic search of the $R$-factor minimum at considerably reduced computational efforts. The strength of this technique becomes particularly evident with more complex structures as is demonstrated for $\mathrm{Ni}(110)-(2 \times 1) \mathrm{O}$ and other systems.
\end{abstract}

\section{Introduction}

The determination of periodic surface structures by low energy electron diffraction (LEED) is usually based on a comparison between measured and calculated intensity/voltage $(I / V)$ spectra for the individual diffracted beams, whereby the degree of agreement is judged by means of evaluating so-called reliability- or $R$-factors. Since the main criterion for the agreement is usually the matching between experimental and theoretical intensity maxima, lengthy calculations of the $I / V$-spectra, typically in steps of $3-5 \mathrm{eV}$, are required for this kind of strategy. If compared with the small set of structural parameters which is derived in this way it appears that a large fraction of intensity data is indeed redundant. This conclusion is supported by general experience from X-ray crystallography, where usually relatively much smaller data sets suffice for precise structural determinations.

The situation with respect to computing efforts becomes even worse if one considers the normal procedure to find the minimum of the $R$-factor which consists in a systematic and independent variation of all structural parameters (grid search technique). If $p$ denotes the number of independent parameters and $n$ the number of data points per parameter (which has to be at least of the order 3-5), then the computational effort increases like $n^{p}$.

Although this strategy is probably the "safest", it is also the most elaborate one, and quite obviously it prevents analysis of more complex structures. Hence the necessity for more sophisticated evaluation schemes is quite obvious.

The present work reports on a twofold strategy for improvement along the sketched lines: At first a novel $R$-factor will be introduced which allows a considerable reduction of the required number of intensity data points by elimination of much of the redundant information quoted above. Secondly, the introduction of an automatic optimisation procedure permits a much more economic search for the $R$-factor minimum. Several other approaches towards this latter goal have been described so far in the literature:

Yang et al. [1] proposed a combination of plausible structural properties with the probability to locate the $R$-factor minima in an exploratory 
grid from where further refinement continues. An automatic search method for localising the $R$-factor minimum has been applied by Powell and de Carvalho [21. This method has the advantage of being generally applicable and independent of the type of $R$-factor by which the distance between the experimental and theoretical $I / V$ spectra is measured. Another very efficient method to speed up the calculation has been developed by Rous ct al. [3] with the tensor LEED approximation. This method allows a very rapid variation of structural parameters by calculating only once a tensor describing the change of $t$-matrices as a function of structure parameters. In the following step a variation of the parameters within certain limits can be performed with little computational effort. The combination of this algorithm with a method to minimise the $R$-factor by the method of the steepest descents has also been recently proposed by Rous et al. [4]. The difference to the method described here lies in the minimisation procedure. In the ways proposed by Powell and de Carvalho [2] and by Rous et al. [4] the steepest descents on the $R$-factor hypersurface are determined after numerically calculating the $R$-factor. In the leastsquares method described below the partial derivatives of the intensity function are required and the deviations from the model structure are analytically calculated. A somewhat different approach has been proposed by Pendry et al. [5-7] by combining the tensor LEED approximation with a "direct" determination of the structural parameters by evaluating the deviations from a model structure with an iteration procedure $[5,6]$ as well as without an iteration procedure using higher order derivatives [7].

With all these procedures still complete $I / V$ spectra are used for comparison between measured and calculated intensity data on the basis of conventional $R$-factors [8-10]. By contrast, the novel $R_{\mathrm{DE}}$-factor is based solely on the comparison of intensities taken at discrete energies. It turned out that the spacing between neighbouring values may be as large as $15-20 \mathrm{eV}$ without any significant loss in reliability of the resulting structural determination. This $R_{\mathrm{DE}^{-} \text {-factor has its roots }}$ in X-ray crystallography where usually only data for a single photon energy are used for analysis. A similar concept has already been proposed for LEED [11,12], but did not find wider applications because of certain shortcomings, such as the under-representation of beams with weak overall intensities and the general problems in determining absolute intensities. These difficulties are overcome in the present approach by proper normalisation rules.

The second stage of improvement consists in the introduction of a well established "leastsquares" optimisation scheme for the search of the minimum of the $R$-factor which combines the advantages of gradient and expansion procedures. The reduction of the computational effort, if compared with the conventional "grid search" technique, becomes more pronounced the more structural parameters are to be determined.

A preliminary short communication on the present work has been published before [13]. In the following a full account on the novel formalism will be given, and its potential will be illustrated with the $\mathrm{O} / \mathrm{Ni}(110)-2 \times 1$ system as an example, whose complete structural analysis (also including conventional LEED analysis procedures) has recently been published elsewhere [14].

\section{The $R_{\mathrm{DE}}$-factor}

There has been a long standing debate about the most appropriate $R$-factor to be used in LEED crystallography. Originally, the comparison bctween experimental and calculated $I / V$-spectra has relied on visual inspection, mainly of the positions of the peak maxima, although in fact actual structure determinations are not making direct use of the energies of intensity maxima.

A prescription for quantitative comparison has been first introduced by Zanazzi and Jona [8], based on the consideration that the maxima of the spectra and overlapping maxima occurring as shoulders of peaks should agree between theory and experiment. Therefore the comparison of derivatives weighted with second derivatives has been taken as criterion. This $R$-factor $\left(R_{Z J}\right)$ has the disadvantage of not being metric, i.e. to be dependent on the range of the energy scale. Nevertheless, $R_{Z J}$ is widely used and leads to reliable 
results. A second concept has been introduced by Pendry [9] using the logarithmic derivatives of the spectra $\left(R_{\mathrm{P}}\right)$. This method basically compares the positions of maxima, a difference in the height of maxima is mostly neglected. The calculation is much faster than that of $R_{\mathrm{ZJ}}$ and is in many respects more advantageous than $R_{\mathrm{ZJ}}$, i.e. it is metric and less sensitive to experimental noise. Therefore $R_{\mathrm{P}}$ has become another standard $R$ factor.

Both the Zanazzi-Jona and Pendry $R$-factors are fairly complicated functions of the (experimental and calculated) intensity data, which cannot be implemented into an optimisation scheme of the type as applied in this work. This can, however, readily be achieved by introducing $R$ factors which are based on the deviation between the normalised experimental and theoretical intensity data, either linearly

$R_{1}=\sum_{i}\left|I_{i}^{\mathrm{ex}}-I_{i}^{\mathrm{th}}\right|$,

or in quadratic form

$R_{2}=\sum_{i}\left|I_{i}^{\mathrm{ex}}-I_{i}^{\mathrm{th}}\right|^{2}$.

In the optimisation scheme to be described below actually $R_{2}$ is minimised, although the final degree of agreement is then quantified in terms of an $R$-factor derived from $R_{1}$. This is done in order to render the results comparable to those of conventional X-ray crystallography where also the linear deviation is used as a standard, denoted as the unweighted $R$-factor. The weighted $R$-factor tak- ing into account of experimental errors, however, is defined as the square root of $R_{2}$. It should be also mentioned that in X-ray crystallography both $R$-factors are usually calculated with the amplitudes, not the intensities. Even more sophisticated fit-functions have been used occasionally in order to suppress the influence of larger deviations which otherwise might dominate the resulting $R$-factor [15]. So far no use of such corrections has been made in the present approach.

The simple $R$-factor $R_{2}$ as defined by eq. (2) had already been used for LEED analysis by Nielsen and Adams [16], but did not find wider application, mainly because it was considered only to be reliable near the minimum. Interestingly, however, it was found in a systematic study by Van Hove et al. [17] that this type of $R$-factor is generally as reliable as others. The linear deviation $R_{1}$ is, on the other hand, implicitly applied in the "direct" method proposed by Pendry [5-7].

The novel $R$-factor is defined as follows

$R_{\mathrm{DE}}=\sum_{g} W_{g} \frac{\sum_{i}\left|J_{i}^{\mathrm{ex}}-C_{g} J_{i}^{\mathrm{th}}\right|}{\sum_{i} J_{i}^{\mathrm{ex}}}$.

First for each beam $g(=h, k)$ the summation $\Sigma_{i}$ is performed over the $n_{g}$ data points at energies $i$, whereby the scaling factor

$C_{g}=\sum_{i} J_{i}^{\mathrm{ex}} / \sum_{i} J_{i}^{\mathrm{th}}$

normalises the absolute intensities $J_{i}$ for each of the individual beams. In this way eventual sys-

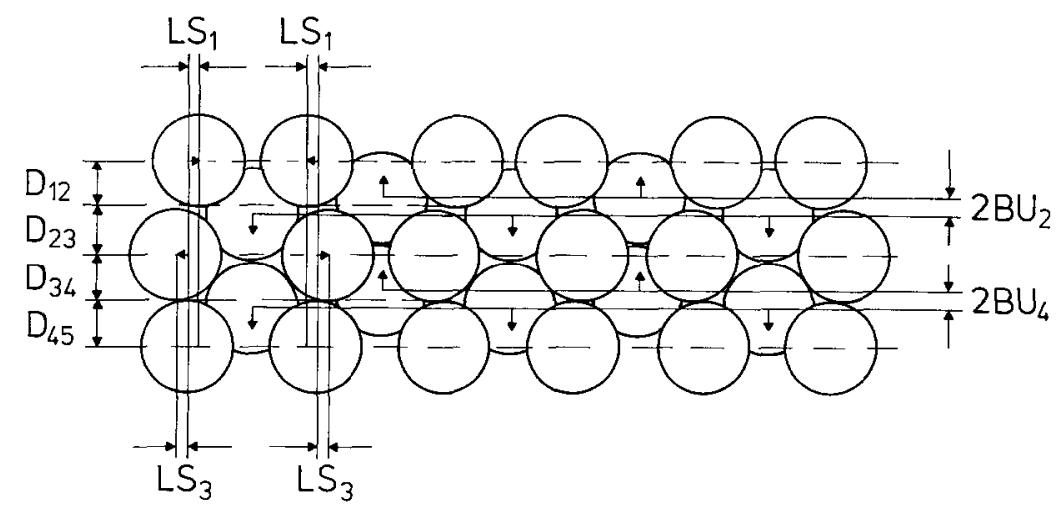

Fig. 1. Model of the II $/ \mathrm{Ni}(110)-1 \times 2$ structure. Cul alung the (110) plane, H atoms omitted. 
tematic experimental errors are eliminated and underestimation of contributions from beams with weak total intensity is avoided.

Next the contributions from each of the beams $g$ are weighted with the factor

$W_{g}=n_{g} / \sum n_{g}$

and then summed up over all beams.

The novel $R_{\mathrm{DE}}$-factor was extensively tested for the $\mathrm{H} / \mathrm{Ni}(110)-1 \times 2$ structure shown in fig. 1 in comparison with the conventional $R_{\mathrm{zJ}^{-}}$and $R_{\mathrm{P}^{-}}$ factors, whereby ordinary energy steps of $3 \mathrm{eV}$ were used for the intensity calculations [13]. (It should be noted that the contributions from the $\mathrm{H}$ atoms to the LEED intensities are completely negligible in this case so that their positions could not be determined [18].) Several of the contour plots calculated as function of some of the structural parameters of this systems were already reproduced in ref. [13]. Generally, the locations of the minima of all three procedures agree to within $\pm 0.02 \AA$ which is commonly regarded as the overall level of accuracy in present LEED structural determinations. The degree of congruence is also reflected by fig. 2 which shows the variation of $R_{\mathrm{P}}, R_{\mathrm{DE}}$ and $R_{\mathrm{ZJ}}$ with the structural parameter LS of the quoted system, that is the lateral shift of $\mathrm{Ni}$ atoms in the topmost atomic layer

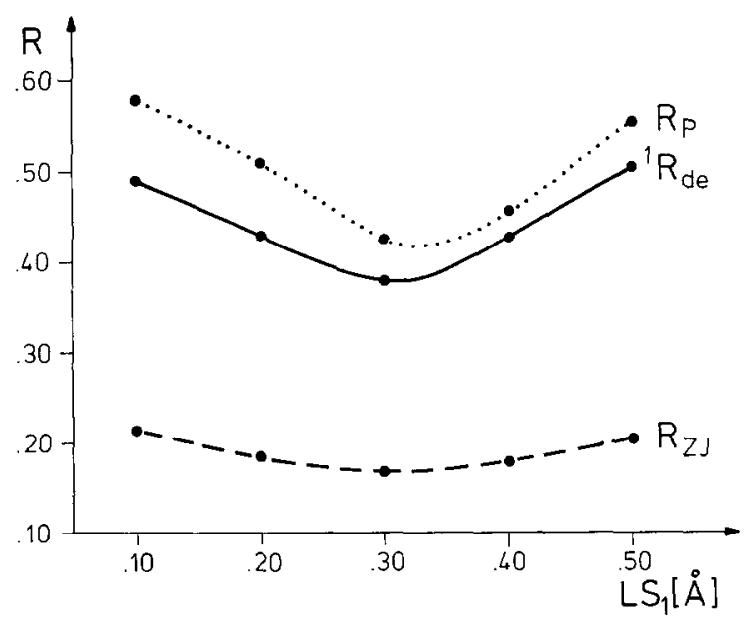

Fig. 2. $R_{\mathrm{P}^{-}}, R_{\mathrm{ZJ}^{-}}$and $R_{\mathrm{DE}^{-}}$factor as a function of the parameter $\mathrm{LS}_{1}$ (see fig. 1). The remaining parameters are kept close to their optimum values.

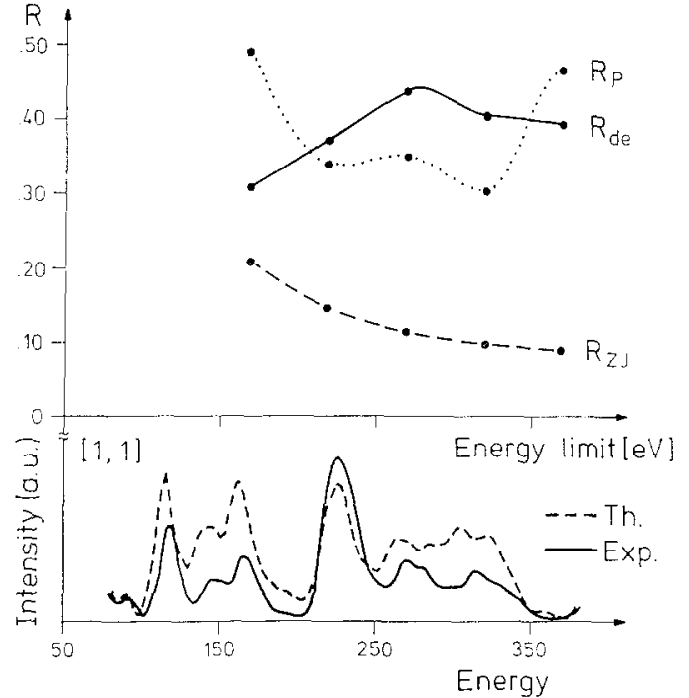

Fig. 3. Dependence of the $R_{\mathrm{p}^{-}}, R_{7 . \mathrm{I}^{-}}$and $R_{\mathrm{DF}}$-factor upon variation of the analysed energy range for the $(1,1)$ beam of $\mathrm{Ni}(110)$.

(with optimal values of the other structural parameters). Again the minima of the three $R$-factors are very close, but also the general shapes of these curves are similar (whereby the numerical values of $R_{\mathrm{DE}}$ are closer to those of $R_{\mathrm{P}}$ ). This demonstrates, that the $R_{\mathrm{DE}}$-factor judges also poorer agreement (i.e. data further away from the minimum) in a similar way as the conventional $R$-factors.

Since $R_{\mathrm{ZJ}}$ is not metric, its minimum value dccreascs monotonically with increasing energy range used for the analysis which might lead to the illusion of a "good" structural determination due to the dominance of high energy peaks. This effect is not present with the metric $R_{\mathrm{P}^{-}}$and $R_{\mathrm{DE}}$-factors, as can be seen from fig. 3 showing data resulting from analysis of the [1,1]-beam of a clean $\mathrm{Ni}(110)$ surface. In these cases variations of the energy range analysed just lead to fluctuations of the numerical value which are different for $R_{\mathrm{r}}$ and $R_{\mathrm{DE}}$ due to their differing definitions.

The subscript "DE" in $R_{\mathrm{DE}}$ denotes "discrete energies" and originates from the mode of selection of the energies for which intensity data are used in the analysis.

After having demonstrated that the novel $R_{\mathrm{DE}}$-factor yields practically identical structural 
data as the $R_{\mathrm{ZJ}^{-}}$and $R_{\mathrm{P}}$-factors if the conventional step width of $3 \mathrm{eV}$ is used, next it was checked how this result becomes affected if the energetic separation of data points is increased. Fig. 4 shows hypersurfaces of $R_{\mathrm{DE}}$ as a function of two of the structural parameters of the Ni(110)$(1 \times 2) \mathrm{H}$ system based on analysis of intensity data taken with varying step width from 11 beams with non-equivalent symmetry. For a narrow step width of $2.5 \mathrm{eV}, 481$ data points are required (fig. 4a). Fig. $4 \mathrm{~b}$ shows the corresponding plot resulting from a step width of $20 \mathrm{eV}$ which is associated with a reduction to only 49 data points. Quite
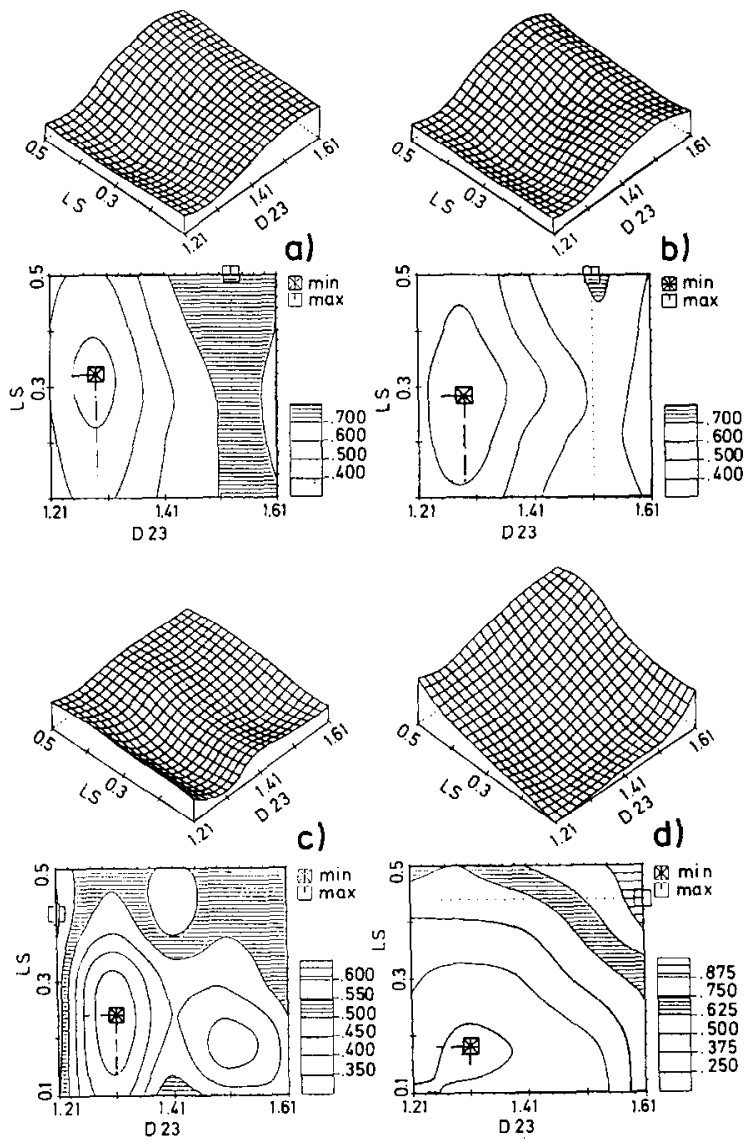

Fig. 4. Perspective views and contour plots of the $R_{\mathrm{DF}}$-factor for variations of the lateral shift $\mathrm{LS}_{1}$ and the layer distance $D_{23}$ of the $\mathrm{H} / \mathrm{Ni}(110)-1 \times 2$ phase (see fig. 1), demonstrating the effect of data reduction: (a) $2.5 \mathrm{eV}$ steps/481 data points; (b) $20 \mathrm{eV}$ steps $/ 49$ data points; (c) $30 \mathrm{eV}$ steps/31 data points; (d) $50 \mathrm{eV}$ steps $/ 12$ data points. Here 11 symmetrically non-equivalent beams have becn analysed.

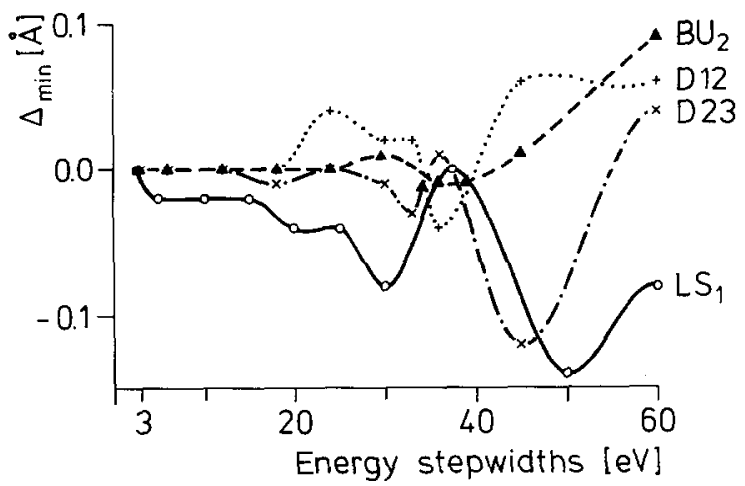

Fig. 5. Deviations of the "best fit" of various structural parameters from their optimum value as a function of the step width on the energy scale. The optimum value was determined with a step density of $3 \mathrm{eV}$ by analysing 480 data points from 11 beams.

remarkably the $R_{\mathrm{DE}}$-minimum is found for almost the same parameters as before. Only further increase of the step width to $30 \mathrm{eV}$ ( 31 data points) or even $50 \mathrm{eV}$ (12 data points) leads to noticeable distortions of the hypersurface so that the $R_{\mathrm{DE}^{-}}$ minimum yields no longer reliable structural information.

Fig. 5 reproduces the deviation $\Delta_{\min }$ of the location of the $R_{\mathrm{DE}}$-factor minimum from the "correct" value (which was determined without data reduction from data with $3 \mathrm{eV}$ energy separation) as a function of the step width for the 5 structural parameters of the $\mathrm{Ni}(110)-(1 \times 2) \mathrm{H}$ system. It becomes evident that essentially the same structural parameters result if the step width is increased even up to $15 \mathrm{eV}$. Quite similar findings were reached for a series of other systems, such as the clean $\mathrm{Ni}(110)$ and $\operatorname{Pd}(110)$ surfaces. That means that the computational effort can safely be reduced by a factor of 5 by this procedure without any significant loss of accuracy.

The upper limit for the tolerable step width will generally be dependent on the total number of data points which will be used for structural analysis. If $n_{p}$ structural parameters are to be determined from $n_{\mathrm{s}}$ diffracted beams, then about $(5-10) \times\left(n_{\mathrm{p}}+n_{\mathrm{s}}\right)$ data points will be sufficient for a reliable structural analysis. $n_{\mathrm{p}}+n_{\mathrm{s}}$ enters here because individual scale factors for each beam have to be treated as independent variables. For 
proper normalisation of intensity data about 5-10 data points per beam will suffice. The stronger one of these structural parameters affects the intensities, the smaller the necessary number of data points (i.e. the larger the step width), as is also reflected by the results of fig. 5. So a reliable determination of the parameter $\mathrm{LS}_{1}$ - in general lateral displacements are known to affect intensity data rather wcakly comparcd to vertical displacements - requires a much larger data base than, for example, the parameter $\mathrm{BU}_{2}$ which has obviously the strongest influence.

It is quite obvious that an increase of the step width up to a critical value mainly eliminates redundant information which is due to the high degree of correlation of the data within an $I / V$ spectrum. These data may essentially be regarded as a superposition of Lorentzians, and hence the data points forming an individual peak are strongly correlated. In addition, also the positions of the $I / V$-maxima with respect to each other are correlated. For this reason it is practically irrelevant which data are selected from the $I / V$-curves, there is no particular significance of the intensity maxima as often believed. This conclusion was extensively checked by shifting the grid of selected intensities. There was also no noticeable difference if instead of constant energetic separations the steps were varied proportional to $\sqrt{V}$, i.e. in constant steps with respect to reciprocal space. The latter prescription is certainly more appropriate but will become only of relevance if data from a much wider energy range than usually taken are to be analysed.

The reduction of the data set does not lead to a significant loss in accuracy as becomes obvious from fig. 6. Here the influence of one parameter on the $I / V$ spectra is illustrated by the difference curves between calculated spectra. As an example the $(0.5,2)$ beam for $\mathrm{O} / \mathrm{Ni}(110)-(2 \times 1)$ is chosen. The parameter, as indicated in the inset of fig. 6 , is the lateral shift of the oxygen. As can be seen its influence on the intensities is approximately linear up to a value of $0.2 \AA$ and changes its sign with approximately the same frequency as maxima in the $I / V$ spectra occur, which are on the average separated by about $15-20 \mathrm{eV}$. It becomes evident that a smaller step width in the $I / V$ analysis

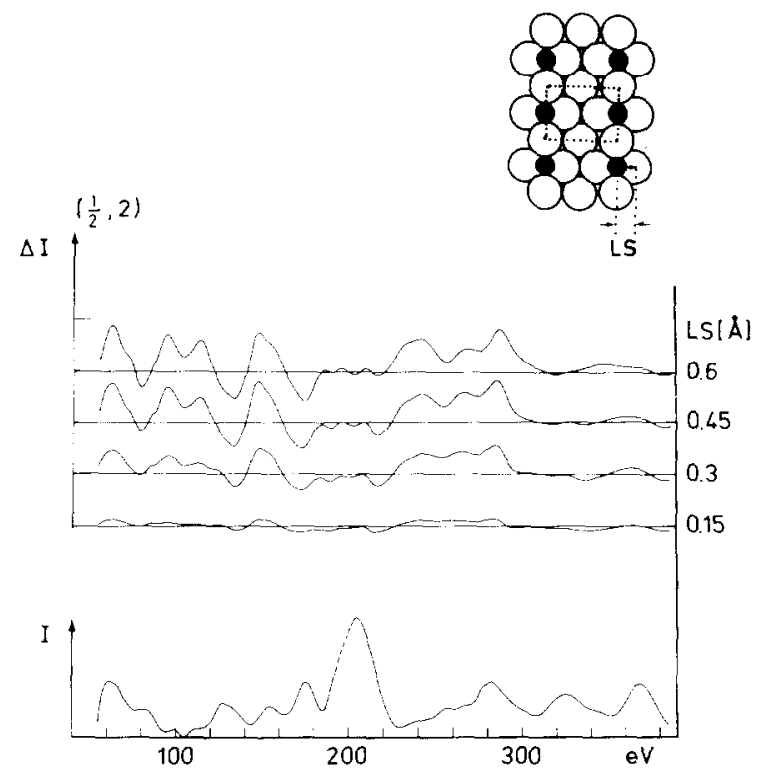

Fig. 6. Calculated $I / V$ curve of the $\left(\frac{1}{2}, 2\right)$ beam of $\mathrm{O} / \mathrm{Ni}(110)$ $(2 \times 1)$ (bottom) and difference spectra for varying the lateral position LS of the $\mathrm{O}$ atom, as indicated in the inset. All curves on same scale.

contains redundant information. It is sufficient to calculate derivatives in steps of about $15-20 \mathrm{eV}$. This once more confirms the conclusion drawn from figs. 4 and 5.

The step width of about $15-20 \mathrm{eV}$ may be also related to the widths of the peaks in the spectrum. The optical potential $V_{\mathrm{j}} \approx 4-5 \mathrm{eV}$ causes peak widths of $2 V_{\mathrm{i}}$ and sufficient intensity is found within a base width of $4 V_{\mathrm{i}}$. This again leads to the conclusion that a smaller step width leads to redundant information. A larger step width, on the other hand, leads to a loss in information. Whether this is tolerable or not depends on the energy range of the spectra and the total number of intensity data as discussed above.

As mentioned before, the novel $R_{\mathrm{DE}}$-factor has its footing in X-ray analysis, where usually intensity data from different beams are analysed only for a single energy. The principle to compare experimental and theoretical intensities at a fixed energy for different beams had been adopted for LEED by Clarke [11]. His $I(g)$-method becomes, however, less reliable if the beams differ from each other considerably with respect to their ab- 
solute intensities. Extended tests revealed indeed clearly the superiority of the $R_{\mathrm{DE}^{-}}$-concept over the previous $I(g)$-method [18].

\section{Optimisation scheme}

The next step towards further reduction of the computational effort consists in the incorporation of an automatic search procedure for finding the minimum of the $R$-factor, i.e. the "best fit" between experimental and calculated intensity data.

Optimisation procedures can in general be divided into two categories, those in which the function which has to be optimised and its derivatives can be calculated throughout the parameter space, and search methods usually applied to problems in which only the function itself can be calculated [19]. The most general and, because of their general applicability, most time consuming methods are search methods where the extrema of a function (here the minimum of the $R$-factor) are localised following a path in the parameter space. The path has to be found by exploratory steps. The method of Hooke and Jeeves [20] which has been applied to LEED by Powell and de Carvalho [2] falls into this latter category.

More economic, if applicable, are in general those methods where the path in the parameter space is found using the derivatives with respect to the parameters. Essentially two main methods can be distinguished, the method of the steepest descent and the expansion method, each having its specific advantages. These optimisation methods are in general much faster than the more general direct search methods and should be well applicable for LEED. The $R$-factor which is usually minimised in structure determinations is an analytic function of the intensities, and the derivatives of the intensities can be calculated either numerically or analytically. The expansion method or the method of the steepest descent therefore seem to be more appropriate than direct search methods. Both methods are applied in X-ray crystallography, the method of the steepest descent usually only in cases where the more time consuming expansion method is not applicable because of a too large number of parameters.
The commonly used $R$-factors, such as $R_{\mathrm{ZJ}}$ or $R_{\mathrm{P}}$, are fairly complex functions of the (experimental and theoretical) intensity data, which cannot be implemented into an optimisation procedure in a simple manner. Hence the first attempts to apply automatic optimisation schemes were based on a direct search procedure [2].

Since the $R_{\mathrm{DE}}$-factor is defined on the basis of the linear deviation, the use of a quantity derived from its square allows incorporation into a leastsquares optimisation scheme in a straightforward manner. More specifically, the minimum of $R_{2}$ (see eq. (2)) with scaling analogous to $R_{\mathrm{DE}}$ (eq. (4)) is used. For the present purpose a nonlinear least-squares fit procedure, which combines the expansion method (near the minimum) with the method of the steepest descent [21], revealed to be particularly efficient. An alternate approach was proposed by Pendry and Heinz [7] which avoids the iterative solutions in the least-squares method by the introduction of higher order derivatives.

The minimum of the $R_{2}$-factor is searched by inserting a linear approximation of the intensity function:

$$
\begin{aligned}
I^{\text {th }}(\boldsymbol{p}) & =I^{\text {th }}\left(\boldsymbol{p}_{0}+\Delta \boldsymbol{p}\right) \\
& =I^{\text {th }}\left(\boldsymbol{p}_{0}\right)+\sum_{j=1}^{k} \frac{\partial I^{\text {th }}\left(\boldsymbol{p}_{0}\right)}{\partial p_{j}} \Delta p_{j}
\end{aligned}
$$

into the minimum condition

$$
\partial R / \partial p_{j}=0(j=1 \ldots k)
$$

which leads to a set of linear equations

$$
\begin{aligned}
& \sum_{i}^{1, n}\left(I_{i}^{\mathrm{ex}}-I_{i}^{\mathrm{th}}\left(\boldsymbol{p}_{0}\right)-\sum_{j=1}^{k} \frac{\partial I_{i}^{\mathrm{th}}\left(\boldsymbol{p}_{0}\right)}{\partial p_{j}} \Delta p_{j}\right) \\
& \quad \times \frac{\partial I_{i}^{\mathrm{th}}\left(\boldsymbol{p}_{0}\right)}{\partial p_{m}}=0 .
\end{aligned}
$$

$\boldsymbol{p}_{0}=\left(p_{1} \ldots p_{n}\right)$ is the set of independent variables and $I_{i}^{\text {th }}, I_{i}^{\text {ex }}$ are the normalised intensities, $I_{i}=$ $W_{g} J_{i} / \Sigma_{i} J_{i}$.

Eq. (7) can be rewritten

$$
\beta_{m}=\sum_{j=1}^{k} \Delta p_{j} \alpha_{j m}
$$


or, in matrix notation:

$\beta=\Delta p \cdot \alpha$,

with

$\beta_{m}=\sum_{i=1}^{n}\left(I_{i}^{\mathrm{ex}}-I_{i}^{\mathrm{th}}\left(\boldsymbol{p}_{0}\right)\right) \frac{\partial I_{i}^{\mathrm{th}}\left(\boldsymbol{p}_{0}\right)}{\partial p_{m}}$

$\alpha_{j m}=\sum_{i=1}^{n}\left(\frac{\partial I_{i}^{\text {th }}\left(p_{0}\right)}{\partial p_{j}} \frac{\partial I_{i}^{\text {ih }}\left(p_{0}\right)}{\partial p_{m}}\right)$.

$\Delta \boldsymbol{p}$ is usually determined solving eq. (8) and iterating the procedure to take account of the non-linearity of the intensity function. The iteration is continued until the improvements in the $R$-factor become smaller than a certain preset limit or the $R$-factor starts to increase again. The inverse of the matrix $\alpha$ is usually called the error matrix, as it is a measure how sensitively the parameters $p_{j}$ influence the result. In the expansion method, eq. (8) is solved by matrix inversion. In the method of the steepest descent, on the other hand, only the diagonal elements $\alpha_{j j}$ are used, which simplifies the calculation.

Both methods have their strengths and deficiencies: Near the minimum with a flat curvature of the $R$-factor function, the gradients become very small and the method of the steepest descent converges rather slowly and may lead to serious errors. Here the expansion method is to be preferred. Far away from the minimum the expansion method, however, does not work properly because non-linear terms are neglected, so that the method of steepest descents has to be preferred. The procedure of Marquardt [21] combines both methods by introducing a parameter $\lambda$, which applies to the diagonal elements of the matrix $\alpha$. In eq. (8) the $\alpha_{j m}$ is replaced by

$\alpha_{j m}^{\prime}=\alpha_{j m}\left(1+\delta_{j m} \lambda\right)$.

$\delta_{j m}$ is the Kronecker symbol. For each individual iteration step the parameter $\lambda$ has to be adjusted by the following scheme: A start value of $\lambda=0.001$ is chosen, if this leads to no improvement in the $R$-factor, $\lambda$ has to be multiplied by 10 . This has to be repeated until the fit proceeds or a preset limit is reached. To minimise the calculational effort, instead a dynamical adjustment of the parameter is chosen:

$\lambda=1+c \sum_{j=1}^{k}\left(\frac{\beta_{j}^{2}}{\alpha_{j j}}\right) \frac{1}{R_{2}}$.

This requires only one calculation per iteration step. The speed of the optimisation can be influenced by a parameter $c$. This parameter is of importance in avoiding too large steps at the beginning of the procedure which might cause the algorithm to localise a false minimum. On the other hand, too small steps would increase the computational effort unnecessarily. Typically a value of $c=0.2$ yields satisfactory convergence properties.

The quoted algorithm of Marquardt was implemented into a standard LEED program. It is organised in a way that the structural parameters may be either varied according to the "grid search" procedure or they are optimised automatically by the fit-procedure. For each iteration step of the automatic optimisation procedure not only the intensities for the actual structure have to be calculated, but also their partial derivatives with respect to the $n$ variable structural parameters, that means in total $(n+1)$ calculations. The applied incremental steps of the structural parameters are $0.02 \AA$ normal to the surface, and $0.05 \AA$ parallel to the surface - corresponding to the current stage of accuracy in LEED crystallography. In the present version of the program all intensities, including those required for determination of the derivatives, are evaluated by fully-dynamic calculations. Further reduction of the computational efforts can probably be achieved by implementing the tensor LEED approximation [13] at this stage, which technique applies a perturbation scheme for evaluating the intensity data for slightly differing model structures and which should therefore be well suited for determination of the derivatives.

\section{Application: the $\mathrm{Ni}(110)-(2 \times 1) \mathrm{O}$ system}

The results of a complete LEED analysis (including application of conventional methods and 
Table 1

Structural parameters for the $\mathrm{Ni}(110)-(2 \times 1) \mathrm{O}$ system in ångström units

\begin{tabular}{ll}
\hline Parameter & Value \\
\hline$Z_{\mathrm{ox}}$ & 0.20 \\
$D_{12}$ & 1.30 \\
$D_{23}$ & 1.23 \\
$D_{34}$ & 1.26 \\
$D_{45}$ & 1.25 \\
$\mathrm{BU}$ & 0.05 \\
$\mathrm{LS}_{\mathrm{ox}}$ & 0.1 \\
$\mathrm{LS}_{\mathrm{Ni}_{z}}$ & 0.0 \\
\hline
\end{tabular}

$R$-factors) has recently been published elsewhere [14]. The resulting structural model is reproduced in fig. 7. In total 8 structural parameters, including relaxation up to the fourth layer, were determined. For the values listed in table 1 the following $R$-factors resulted: $R_{\mathrm{ZJ}}=0.09, R_{\mathrm{P}}=0.18, R_{\mathrm{DE}}=$ 0.22 . This has to be considered as excellent agreement. The structure is of the "missing row" type with the $\mathrm{O}$-atoms located in slightly asymmetric bridge sites. The lateral displacement of the $\mathrm{O}$ atoms out of symmetric bridge sites provides only a rather weak effect on the $R$-factor, but is supported by symmetry considerations following from HREELS experiments [22]. The lateral shift $\mathrm{LS}_{o x}$ is not shown in fig. 7 and is not included in the optimisation described below. In the following this system will be used to illustrate the potential
Table 2

Data set for the LEED analysis of the $\mathrm{O} / \mathrm{Ni}(110)-2 \times 1$ phase. (106 data points were taken from 8 systematically non-equivalent beams at $15 \mathrm{eV}$ energy steps)

\begin{tabular}{rllllllll}
\hline $\begin{array}{l}\text { Energy } \\
(\mathrm{eV})\end{array}$ & \multicolumn{1}{l}{ Beam } & & & & & \\
\cline { 2 - 7 } & $(1,1)$ & $(1,0)$ & $(0,1)$ & $(0,2)$ & $(1,2)$ & $\left(\frac{1}{2}, 0\right)$ & $\left(\frac{1}{2}, 1\right)$ & $\left(\frac{1}{2}, 2\right)$ \\
\hline 40.0 & & & $\mathrm{X}$ & & & $\mathrm{X}$ & $\mathrm{X}$ & \\
55.0 & & & $\mathrm{X}$ & & & $\mathrm{X}$ & $\mathrm{X}$ & \\
70.0 & & $\mathrm{X}$ & $\mathrm{X}$ & & & $\mathrm{X}$ & $\mathrm{X}$ & \\
85.0 & $\mathrm{X}$ & $\mathrm{X}$ & $\mathrm{X}$ & & & $\mathrm{X}$ & $\mathrm{X}$ & \\
100.0 & $\mathrm{X}$ & $\mathrm{X}$ & $\mathrm{X}$ & & & $\mathrm{X}$ & $\mathrm{X}$ & \\
115.0 & $\mathrm{X}$ & $\mathrm{X}$ & $\mathrm{X}$ & $\mathrm{X}$ & & & $\mathrm{X}$ & $\mathrm{X}$ \\
130.0 & $\mathrm{X}$ & $\mathrm{X}$ & $\mathrm{X}$ & $\mathrm{X}$ & & & $\mathrm{X}$ & $\mathrm{X}$ \\
145.0 & $\mathrm{X}$ & $\mathrm{X}$ & $\mathrm{X}$ & $\mathrm{X}$ & $\mathrm{X}$ & & $\mathrm{X}$ & $\mathrm{X}$ \\
160.0 & $\mathrm{X}$ & $\mathrm{X}$ & & $\mathrm{X}$ & $\mathrm{X}$ & & $\mathrm{X}$ & $\mathrm{X}$ \\
175.0 & $\mathrm{X}$ & $\mathrm{X}$ & & $\mathrm{X}$ & $\mathrm{X}$ & & $\mathrm{X}$ & $\mathrm{X}$ \\
190.0 & $\mathrm{X}$ & $\mathrm{X}$ & & $\mathrm{X}$ & $\mathrm{X}$ & & $\mathrm{X}$ & $\mathrm{X}$ \\
205.0 & $\mathrm{X}$ & $\mathrm{X}$ & & $\mathrm{X}$ & $\mathrm{X}$ & & $\mathrm{X}$ & $\mathrm{X}$ \\
220.0 & $\mathrm{X}$ & $\mathrm{X}$ & & $\mathrm{X}$ & $\mathrm{X}$ & & $\mathrm{X}$ & $\mathrm{X}$ \\
235.0 & $\mathrm{X}$ & $\mathrm{X}$ & & $\mathrm{X}$ & $\mathrm{X}$ & & $\mathrm{X}$ & $\mathrm{X}$ \\
250.0 & $\mathrm{X}$ & $\mathrm{X}$ & & $\mathrm{X}$ & $\mathrm{X}$ & & $\mathrm{X}$ & $\mathrm{X}$ \\
265.0 & $\mathrm{X}$ & $\mathrm{X}$ & & $\mathrm{X}$ & $\mathrm{X}$ & & & $\mathrm{X}$ \\
280.0 & $\mathrm{X}$ & $\mathrm{X}$ & & $\mathrm{X}$ & $\mathrm{X}$ & & & $\mathrm{X}$ \\
295.0 & $\mathrm{X}$ & $\mathrm{X}$ & & $\mathrm{X}$ & $\mathrm{X}$ & & & $\mathrm{X}$ \\
310.0 & $\mathrm{X}$ & $\mathrm{X}$ & & $\mathrm{X}$ & $\mathrm{X}$ & & & $\mathrm{X}$ \\
340.0 & $\mathrm{X}$ & $\mathrm{X}$ & & $\mathrm{X}$ & $\mathrm{X}$ & & $\mathrm{X}$ \\
\hline
\end{tabular}

of the novel procedure described in the present work.

The analysis was based on experimental intensity data from 8 beams taken at normal incidence within an energy range between 40 and $340 \mathrm{eV}$

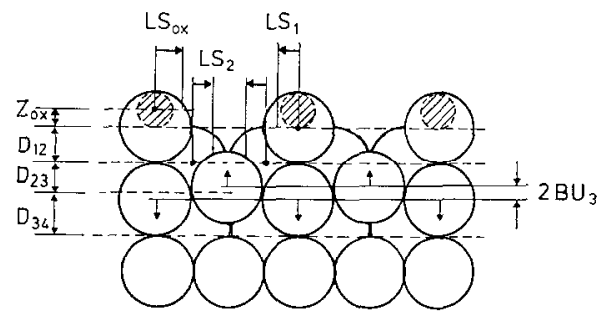

a

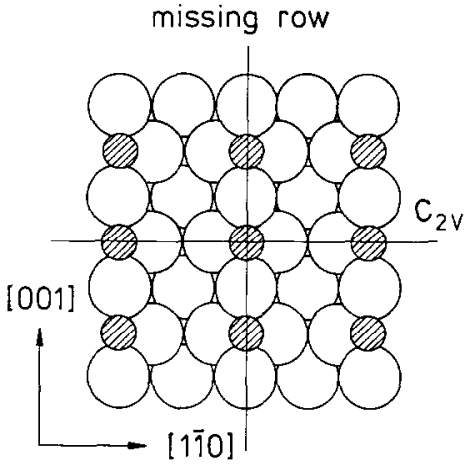

b

Fig. 7. Structural model and parameters varied in the analysis of the "missing row" model for the $\mathrm{O} / \mathrm{Ni}(110)-2 \times 1 \mathrm{phase}$. Left panel: cut along (001) plane; right panel: topview. Arrows indicate the shift in the topmost laycrs with respect to the bulk positions. 
Table 3

Fit table ( $R_{\mathrm{DF}}$-factor and corresponding structural parameters for each iteration step) for the application of the optimization scheme to $\mathrm{O} / \mathrm{Ni}(110)-2 \times 1$, analysing the intensity data listed in table 2 (the fit starts from an undistorted "missing row" structure with oxygen assumed $0.3 \AA$ above the topmost $\mathrm{Ni}$ layer)

\begin{tabular}{llllllll}
\hline Iteration & $R_{\mathrm{de}}$ & $Z_{\text {ox }}(\AA)$ & $\mathrm{BU}(\AA)$ & $D_{12}(\AA)$ & $D_{23}(\AA)$ & $D_{34}(\AA)$ & $D_{45}(\AA)$ \\
\hline 0 & 0.388 & 0.300 & 0.000 & 1.246 & 1.246 & 1.246 & 1.246 \\
1 & 0.314 & 0.235 & 0.017 & 1.255 & 1.252 & 1.257 & 1.242 \\
2 & 0.274 & 0.227 & 0.036 & 1.265 & 1.252 & 1.251 & 1.240 \\
3 & 0.251 & 0.253 & 0.051 & 1.282 & 1.240 & 1.249 & 1.246 \\
4 & 0.248 & 0.256 & 0.054 & 1.290 & 1.228 & 1.255 & 1.248 \\
5 & 0.247 & 0.244 & 0.054 & 1.293 & 1.226 & 1.262 & 1.248 \\
6 & 0.242 & 0.231 & 0.053 & 1.294 & 1.225 & 1.264 & 1.247 \\
7 & 0.241 & 0.220 & 0.053 & 1.293 & 1.223 & 1.264 & 1.247 \\
\hline
\end{tabular}

and selected for step widths of $15 \mathrm{eV}$. In total 106 intensity data were used as listed in table 2. The calculations were performed by using the following non-structural parameters: A uniform Debye temperature (for substrate as well as adsorbate atoms) of $250 \mathrm{~K}$ was assumed. Up to 8 phase shifts for $\mathrm{O}$ and 9 phase shifts for Ni were used. The crystal potential for $\mathrm{Ni}$ was obtained from a superposition of free atomic potentials, the overlap was taken into account by a Mattheiss procedure. The phase shifts for $\mathrm{O}$ were taken from the literature [23]. The real part of the inner potential was taken as $V_{\text {or }}=-10 \mathrm{eV}$, while its imaginary part was set as energy-dependent

$V_{\mathrm{oi}}=0.85\left(V+V_{\mathrm{or}}\right)^{1 / 3}$.

The bulk scattering matrices were calculated using the layer doubling scheme.

The efficiency of the novel technique is demonstrated by the following results: Starting from the "missing row" model exhibiting structural param- eters of bulk Ni plus an (arbitrary) vertical position of the $\mathrm{O}$-atom of $Z_{\mathrm{ox}}=0.3 \AA$, simultaneous refinement of 6 structural parameters by means of the automatic least-squares optimisation scheme was performed on the basis of intensities with 15 $\mathrm{eV}$ step widths. As can be seen from table 3, the $R_{\text {DE }}$ factor decreases continuously from iteration to iteration, and reaches its minimum after 7 steps. The resulting structural parameters are practically identical to those from application of the grid search technique as listed in table 1 . In principle, the automatic least-squares procedure is expected to lead to even more reliable results than the grid search technique, since it refines all structural parameters really simultaneously and is not limited by a preset parameter mesh.

In order to check if the resulting structure is indeed independent of the starting configuration, another series of calculations was performed in which different values for the parameters $\mathrm{BU}, D_{23}$ and $D_{34}$ had been chosen. In addition, the param-

Table 4

Comparison of the structural result of two different optimization runs

\begin{tabular}{llllllllll}
\hline & $R_{\mathrm{de}}$ & $Z_{\mathrm{ox}}$ & $\mathrm{BU}$ & $\mathrm{LS}_{\mathrm{Ni}_{2}}$ & $D_{12}$ & $D_{23}$ & $D_{34}$ & $\overline{D_{45}}$ & $\Delta V_{\mathrm{or}}[\mathrm{eV}]$ \\
\hline$S_{\mathrm{A}}$ & 0.388 & 0.300 & 0.000 & - & 1.246 & 1.246 & 1.246 & 1.246 & - \\
$S_{\mathrm{B}}$ & 0.630 & 0.300 & 0.050 & 0.000 & 1.246 & 1.296 & 1.296 & - & 0.000 \\
$E_{\mathrm{A}}$ & 0.241 & 0.220 & 0.053 & - & 1.293 & 1.223 & 1.264 & 1.247 & - \\
$E_{\mathrm{B}}$ & 0.228 & 0.206 & 0.052 & 0.011 & 1.295 & 1.226 & 1.260 & - & -1.1 \\
\hline
\end{tabular}

Run A: $40-340 \mathrm{eV}, 15 \mathrm{eV}$ steps, 106 data points. Run B: $40-380 \mathrm{eV}, 15 \mathrm{eV}$ steps, 120 data points. $S_{\mathrm{A}}$ and $S_{\mathrm{B}}$ denote the parameter sets at the start of the respective runs, $E_{\mathrm{A}}$ and $E_{\mathrm{B}}$ the end configurations, respectively. 
eter $\mathrm{LS}_{\mathrm{Ni}_{2}}$ and the real part of the inner potential, $V_{\text {or }}$, were included as further adjustable parameters. As can be seen from the comparison with the previous result (table 4 ), practically the identical structure is obtained. The $R_{\mathrm{DE}}$-factor is slightly improved due to optimisation of $V_{\text {or }}$ to a value of $-11.1 \mathrm{eV}$.

The stronger the influence of a parameter on the intensities, the more it will also affect the value of the $R$-factor and the precision of the structural determination. This can be quantified in terms of the curvature of the $R$-factor with respect to the chosen parameter in the region of the global minimum. As mentioned, the error matrix - a byproduct of the fit procedure - contains this information. It turns out that for the parameter $\mathrm{BU}$ this is, for example, about 5 times as large as for $Z_{\mathrm{ox}}$, and 30 times as large as for $\mathrm{LS}_{\mathrm{Ni}_{2}}$ ! The relative insensitivity to the position of the oxygen atoms is due to their small scattering cross sections, their lateral shift, $\mathrm{LS}_{\mathrm{ox}}$, is for this reason also hardly influencing $R_{\mathrm{DE}}$ at all. $\mathrm{LS}_{\mathrm{ox}}=0.1$ was slightly improving the $R_{\mathrm{DE}}$-factor; but an asymmetric position of the $\mathrm{O}$-atom found its true justification only on the basis of the quoted HREELS results [22].

\section{Discussion}

The procedure for LEED crystallography presented in this work leads to substantial reduction of the calculation efforts and hence enables also analysis of more complex structures, first because of the use of a considerably smaller set of intensity data and secondly because of the introduction of an economic evaluation scheme for the search of the $R$-factor minimum in a multi-dimensional parameter space.

The first aspect concerns the use of intensity data only at discrete energies instead of continuous $I / V$-spectra. As was demonstrated, an increase of the step width to about $15 \mathrm{eV}$ is without any significant effect on the degree of accuracy for the resulting structural determination. This has to be attributed to the fact that, due to the physical origin of the LEED intensity data, their values at different energies are strongly correlated which renders a substantial fraction of the information contained in continuous $I / V$-spectra in fact redundant. The stated increase of the step width from $3 \mathrm{eV}$ in conventional LEED analysis to 15 $\mathrm{eV}$ yields a reduction of the computational effort by a factor of 5 without any significant loss of structural information.

An even more pronounced effect is reached by application of the described optimisation scheme for searching the minimum of the $R$-factor, particularly in the case of more complicated structures requiring a larger set of structural parameters. If $p$ is the number of the latter and $n$ the number of data points per parameter (which has at least to be 3 ), then with the conventional grid search procedures simultaneous refinement of the $p$ parameters requires evaluation of

$M_{\mathrm{gs}}=n^{p}$

structural models.

The introduction of the novel $R_{\mathrm{DE}}$-factor, on the other hand, which is simply based on the difference between experimental and calculated intensity data, enables the application of a nonlinear "least-squares" optimisation scheme as a much more economic way towards the $R$-factor minimum. Now only

$M_{\mathrm{ls}}=(p+1) N$

structural models have to be evaluated, where $N$ is the number of iteration steps. The Marquardt algorithm applied here for searching the minimum

Table 5

Comparison of the calculational effort for the grid search method and the least squares method

\begin{tabular}{|c|c|c|c|c|c|}
\hline \multirow[t]{3}{*}{ System } & \multicolumn{3}{|c|}{ Search procedure } & \multirow{2}{*}{\multicolumn{2}{|c|}{$\begin{array}{l}\text { Least squares } \\
\text { method }\end{array}$}} \\
\hline & \multirow[t]{2}{*}{$p$} & \multicolumn{2}{|l|}{$M_{\mathrm{gs}}$} & & \\
\hline & & $n=3$ & $n=5$ & $N$ & $M_{\mathrm{ls}}$ \\
\hline $\mathrm{Ni}(110)$ & 3 & 27 & 125 & 6 & 24 \\
\hline $\mathrm{Ni}(110)$ & 4 & 81 & 625 & 7 & 35 \\
\hline $\mathrm{Ni}(110)-(2 \times 1) \mathrm{O}$ & 6 & 729 & 15625 & 7 & 56 \\
\hline $\mathrm{Ni}(110)-(1 \times 2) \mathrm{H}$ & 8 & 6561 & 390625 & 10 & 90 \\
\hline
\end{tabular}

$p$ : number of free parameters, $n$ : number of data points per parameter, $N$ : number of iterations after which the minimum had been reached, $M_{\mathrm{gs}}, M_{1 \mathrm{~s}}:$ number of structural models. 
of the $R_{\mathrm{DF}}$-factor combines the advantages of the expansion method with those of the method of steepest descent and exhibits excellent convergence criteria, that means $N$ becomes rather small as becomes evident from the actual example presented in section 4 .

A detailed comparison for various structures analysed is made in table 5 . It lists the number $p$ of structural parametcrs to be refincd, the number of model structures $M_{\mathrm{gs}}$ to be evaluated by application of the search method with $n=3$ or 5 data points per parameter, as well as the number of iteration steps $N$ required with the present "least squares" optimisation scheme and the resulting number $M_{1 \mathrm{~s}}$ of model structures to be evaluated. The power of the novel procedure becomes clearly evident, particularly from inspection of the last row: For a system with 8 structural parameters the computational effort is reduced by more than a factor of 70 with respect to that needed for application of a (crude) grid search technique. If this is combined with the effect of the smaller set of intensity data as outlined above, an overall reduction in computational effort by a factor of about 400 results without any loss in precision of the structural determination! It becomes, in addition, evident from inspection of table 4 that structural analysis on the basis of "grid search" techniques will become a rather hopeless task for systems with more than about 6 parameters which have to be refined independently.

The present procedure has its roots in X-ray crystallography so that also some comparisons with the experience from this much more developed field is in place: In determinations of bulk structures based on X-ray diffraction usually only a single energy (wavelength) is applied for which the intensities are analysed. The best $R$-factors to be achieved reach down to values as small as 0.02 to 0.05 . In these cases, however, a number of non-structural parameters, such as absorption and anisotropic temperature factors, have to be corrected very carefully. If only overall isotropic temperature coefficients are included (as is the case with all LEED structural determination) usually at best minimum $R$-factors of 0.1 to 0.2 are obtained which are in the same range as the values which can he reached by LEED crystallography. Struct- ural parameters determined by assuming isotropic thermal vibrations can be uncertain up to about $0.05 \AA$, which can be suppressed by about one order of magnitude by inclusion of anisotropic temperature coefficients. One may conclude that anisotropic vibrations can cause similar uncertainties in the case of LEED. At present this influence cannot be estimated, since a practical method to include anisotropic vibrations in the multiple scattering formalism is not yet available. The frequently quoted error limit of $0.02 \AA$ in LEED studies does not include systematic errors due to shortcomings of the theory.

The $R$-factor is not necessarily the best criterion to judge the quality of a structural analysis. In X-ray crystallography even more reliable results are obtained by applying the $\chi^{2}$ test in which deviations between measured and calculated intensity data are weighted by the uncertainty of the experimental data due to the counting statistics (after elimination of all systematic errors such as misorientation, absorption correction etc.). Such a procedure is so far, however, not yet applicable in LEED crystallography where the degree of precision of experimental data is still limited by systematic experimental errors.

\section{References}

[1] W.S. Yang, F. Jona and P.M. Marcus, J. Vac. Sci. Technol. B 1 (1983) 718.

[2] P.G. Powell and V.E. de Carvalho, Surf. Sci. 187 (1987) 175.

[3] P.J. Rous, J.B. Pendry, D.K. Saldin, K. Heinz, K. Müller and N. Bickel, Phys. Rev. Lett. 57 (1986) 2951.

[4] P.J. Rous, M.A. Van Hove and G.A. Somorjai, Surf. Sci. 226 (1990) 15.

[5] J.B. Pendry, K. Heinz and W. Oed, Proc. 11th Int. Vacuum Congress Köln, 1989, Vacuum 41 (1990) 340.

[6] J.B. Pendry, K. Heinz and W. Oed, Phys. Rev. Lett. 61 (1988) 2953.

[7] J.B. Pendry and K. Heinz, Surf. Sci. 230 (1990) 137.

[8] E. Zanazzi and F. Jona, Surf. Sci. 62 (1977) 1.

[9] J.B. Pendry, J. Phys. C 13 (1980) 937.

[10] J. Philip and J. Rundgren, in: Determination of Surface Structures by LEED, Eds. P.M. Marcus and F. Jona (Plenum, New York, 1984).

[11] (a) L.J. Clarke, Surface Crystallography - An Introduction to Low Energy Electron Diffraction (Wiley, Chichester, 1985);

(b) L.J. Clarke, Vacuum 29 (1979) 405 
[12] Y. Gauthier, R. Baudoing, Y. Joy, C. Gaubert and J. Rundgren, J. Phys. C 17 (1984) 4547.

[13] G. Kleinle, W. Moritz, D.L. Adams and G. Ertl, Surf. Sci. 219 (1989) L637.

[14] G. Kleinle, J. Wintterlin, G. Ertl, R.J. Behm, F. Jona and W. Moritz, Surf. Sci. 225 (1990) 171.

[15] See for example: E. Prince, Mathematical Techniques in Crystallography and Materials Science, (Springer, New York, 1982).

[16] H.B. Nielsen and D.L. Adams, J. Phys. C15 (1982) 615.

[17] M.A. Van Hove and R.J. Koestner, in: Determination of Surface Structures by LEED, Eds. P.M. Marcus and F. Jona (Plenum, New York, 1984) p. 357.
[18] G. Kleinle, Thesis, FU Berlin (1989).

[19] See for example: G.R. Walsh, Methods of Optimization (Wiley, Chichester, 1985).

[20] R. Hooke and F.A. Jeeves, J. Am. Chem. Soc. 8 (1961) 212.

[21] D.W. Marquardt, J. Soc. Ind. Appl. Math. 11 (1963) 431.

[22] B. Voigtländer, S. Lehwald and H. Ibach, Surf. Sci. 225 (1990) 162.

[23] S.Y. Tong, A. Maldonado, L.H. Li and M.A. Van Hove, Surf. Sci. 186 (1987) 45. 